\title{
QoS Based Evaluation of Multipath Routing Protocols in Manets
}

\author{
Saman Shakir ${ }^{1, *}$, Samiullah Khan ${ }^{2}$, Liaq Hassain ${ }^{1}$, Matiullah ${ }^{3}$ \\ ${ }^{1}$ Department of Computer Science, University of Engineering and Technology, Peshawar, Pakistan \\ ${ }^{2}$ Faculty of Computing, Capital University of Science and Technology, Islamabad, Pakistan \\ ${ }^{3}$ Department of Basic Sciences and Islamyat, University of Engineering and Technology, Peshawar, Pakistan
}

\section{Email address:}

samikhan.1982@gmail.com (S. Khan),saman.itec@gmail.com (S. Shakir), laiqhasan@uetpeshawar.edu.pk (L. Hassan), matiullah28618@yahoo.com (Matiullah)

${ }^{*}$ Corresponding author

\section{To cite this article:}

Saman Shakir, Samiullah Khan, Liaq Hassain, Matiullah. QoS Based Evaluation of Multipath Routing Protocols in Manets. Advances in Networks. Vol. 5, No. 2, 2017, pp. 47-53. doi: 10.11648/j.net.20170502.13

Received: October 1, 2017; Accepted: October 16, 2017; Published: December 7, 2017

\begin{abstract}
Mobile Ad Hoc Networks (MANETs) are wireless networks formed by several nodes communicating on a peer-to-peer basis without being connected to any fixed infrastructure. These nodes could be laptop computers, personal digital assistants, mobile phones or sensors dispersed in an area to measure certain data and send the information to a larger node. Where a source node and a destination node are not within direct range, they communicate through multi-hop routing, i.e. nodes in between them relay messages between source and destination. The routing protocol plays a key role in finding and maintaining the route in MANETs. Routing protocol can be a uni-path and multi-path. A multipath routing protocol is designed to increase the reliability in MANET. This research work focuses on Quality of Service (QoS) based evolution of multipath routing protocol. For this purpose, diverse type of simulation scenarios is designed to find the impact of mobility, increasing the number of nodes and pause time in MANETs. The results revealed that multipath routing protocol has comparatively less delay (percentage decrease of $81.52 \%$ ). While unipath routing protocols have less packet drop ratio (percentage decrease of $47.78 \%$ ) and routing overhead (percentage decrease of $99.30 \%$ ).
\end{abstract}

Keywords: MANETs, Unipath Routing Protocols, Multipath Routing Protocols, Mobility, Traffic Source

\section{Introduction}

Mobile Ad hoc Networks (MANETs) is the type of Ad hoc Networks; It is the collection of nodes, i.e. mobiles, laptops, etc. which do not require any fixed physical infrastructure, i.e. access points. Each node act as a source, destination or a relay station, which is used to send data to the destination. MANETs are self-organizing, self-distributing and selfmaintaining. MANETs can be used in many applications, i.e. Home Networks, Military application, Disaster relief operation and Civilian environment [1]. In spite of all these applications, there are some challenges while working with MANETs such as autonomous and infrastructure less, multihop routing, mobility, topology and limited battery time.

Routing is the forwarding of packets within the ad hoc network towards ultimate destinations. [2]. There are two types of routing protocols, i.e. unipath and multipath routing protocols. Unipath routing protocols use single path while multipath routing uses multiple paths for data transmission. Multipath routing can be classified into three types proactive, reactive and hybrid as shown in figure 1 [3].

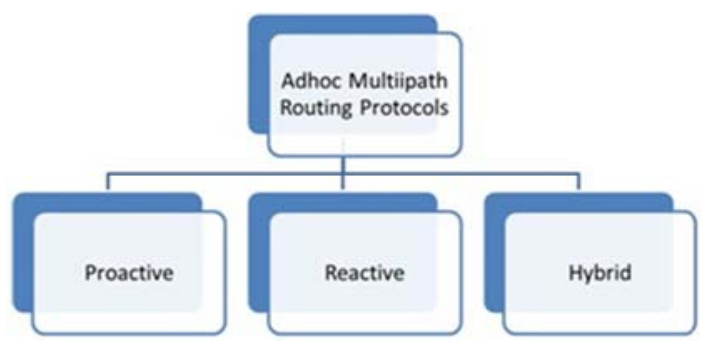

Figure 1. Classification of multipath routing protocols in MANETs (Tarique et al., 2009).

In Proactive Multipath Routing, a routing table is prepared for each node whether there is a requirement to send data or 
not, while in reactive routing path is established when needed only. Reactive routing is composed of two parts:

Route discovery is done when a new path is needed to send data to a destination, RREQ packets are flooded to all the neighbours of the source and RREP is sent to the source through the route reversal process or piggy backing from the destination.

Route maintenance is the process to maintain the paths or to detect the path failures, RERR is generated and sent to the source if the path is broken and hence again a route discovery process is created to establish a new path.

\section{Literature Review}

Routing in MANETs is an immense and interesting topic of research nowadays. Unipath and multipath routing are described, and their difference is evaluated in many research papers. In this research paper, two most used unipath routing protocol analyzed are Dynamic Source Routing (DSR) and Ad hoc On-Demand Distance Vector (AODV) [13].

DSR is a unipath routing protocol basically used for multihop routing. As the name of this protocol shows, it is based on source routing. When a node wants to send data to the destination, it checks the cache as every node has the route cache. If the path is available, it starts sending data. Otherwise, it initiates a route discovery process by sending RREQ packets to all the neighbouring nodes and when a path is established as the destination sends a RREP to the source. This path is maintained and checked throughout by the process of route maintenance [4]. Mechanism of DSR is elaborated in Figure 2:

Multipath Dynamic Address Routing (M-DART) is the proactive multipath protocol which is the enhancement of Dynamic Address Routing (DART) protocol and is based on the Distributed hash table (DHT) whose basic function is to spread the location of node throughout the node [7].

In MANETs, data is sent from source to destination, but the point is to send that data efficiently without causing much delay and in proper order. For evaluating MANETs, there are three performance evaluation techniques, i.e. measurement, analytical modelling and simulations.

There is a need for QoS based evolution of multipath routing protocols using mobility, network density and pause time [11].

\section{Simulation Scenarios}

Mobility, a number of nodes and pause time are the important factors that affect the performance of unipath and multipath routing protocols. In view of this, three main simulation scenarios are designed, i.e. increasing traffic sources, mobility and pause time. Each simulation has three more sub-scenarios in order to thoroughly study the impact of these factors on unipath and multipath routing protocols.

\subsection{Increasing Traffic Source}

In these scenarios, different numbers of nodes are used with same mobility speed $(1.4 \mathrm{~m} / \mathrm{sec})$ and pause time $(2$ seconds). The average packet drop rate of the unipath and multipath routing protocols are compared while increasing the traffic sources from 5 to 15 nodes while keeping the mobility and the pause time constant.

\subsection{Increasing Mobility}

In mobility scenarios, the speed of mobile nodes is chosen with respect to standard walking speed $(1.4 \mathrm{~m} / \mathrm{sec})$, running speed $(3.3 \mathrm{~m} / \mathrm{sec})$ and vehicle speed $(11.11 \mathrm{~m} / \mathrm{sec})$. Other parameters such as a number of nodes and pause time are kept same. Unipath and multipath routing protocols are compared with increasing the mobility from $1.4 \mathrm{~m} / \mathrm{Sec}$ to $11.11 \mathrm{~m} / \mathrm{Sec}$ [12].

\subsection{Pause Time}

In pause time scenarios, the pause time kept varying for each scenario such as 2, 5 and 10 seconds while other parameters are kept same such as mobility speed and number of nodes. Analyzing average packet drop rate for the unipath and multipath routing protocols by varying the pause time from 2 to $10 \mathrm{Sec}$. In each sub-scenario, i.e. $2 \mathrm{sec}, 5 \mathrm{Sec}$ and $10 \mathrm{sec}$ average packet drop rate for all the protocols are observed, and the result shows that DSR has the least average packet drop rate. In addition to this, the detail parameters configuration are mentioned in table 1 .

\subsection{Performance Analysis Parameters}

The following parameters are used for performance analysis of multipath routing protocols.

\subsubsection{Average Packet Drop Rate}

Time for packets for reaching from a source to destination.

Average Packet Drop Rate $=\frac{\sum_{i=1}^{n} \text { Packet_Drop }_{i}}{\text { Simulation_Time }(\text { Seconds })_{1}}(1)$

Table 1. General simulation parameters configurations.

\begin{tabular}{ll}
\hline Parameter & Values \\
\hline Simulator & NS-2 (Version 2.34) \\
Channel type & Channel/Wireless channel \\
Radio-propagation model & Propagation/Two ray round wave \\
Network interface type & Phy/Wireless Phy \\
MAC Type & Mac /802.11 \\
Interface queue Type & Queue/Drop Tail \\
Link Layer Type & LL \\
Antenna & Antenna/Omni Antenna \\
Maximum packet in buffer & 60 \\
Unipath & AODV, DSR \\
Multipath & AOMDV, M-DART \\
Area (m ${ }^{2}$ ) & 800 x 800 \\
Traffic Type & CBR/UDP \\
Simulation Time & 300 seconds (5 Minutes) \\
\hline
\end{tabular}

\subsubsection{Average Per Packet Delay}

Packet delay is the total time that a packet consumes in travelling from a source to destination. Packet delay may be due to route discovery, queuing, propagation and transmission delay. 


$$
\text { Average Per Packet Delay }=\frac{\sum_{i=1}^{n}\left(\text { Receive_Time }_{i}-\text { Send_Time }_{i}\right)}{\text { Total Number of Packets }(n)}
$$

\subsubsection{Average Normal Routing Load}

Data traffic related to the routing updates are normal routing load. Mathematically it can be shown as mentioned below.

$$
\text { Average Normal Routing Load }=\frac{\sum_{i=1}^{n} \text { Route_Pkt }_{i}}{\text { Simulation_Time(Seconds })}
$$

\section{Result and Discussion}

Different simulators are used for the simulation purpose of protocols like NS-2, NS-3, Qualnet, OPNET and OMNet ++. In this research, NS-2 is used as it is the most commonly used discrete event simulator for simulating unipath and multipath routing protocols. NS-2 is chosen for this research because of its free, open source and easy to understand [10]. The NS-2 General configuration for this research is described in table 1.

\subsection{Traffic Source}

Figure 2 shows the impact of the average packet drop rate. DSR has the least packet dropped rate. Hence Unipath routing protocol outperforms multipath routing protocol with respect to packet dropped rate. DSR maintains the soft route. These soft routes make DSR very robust due to the facts that DSR maintains multiple routes to a single destination using single route discovery method. DSR uses the second route in case of first route failure and so on.

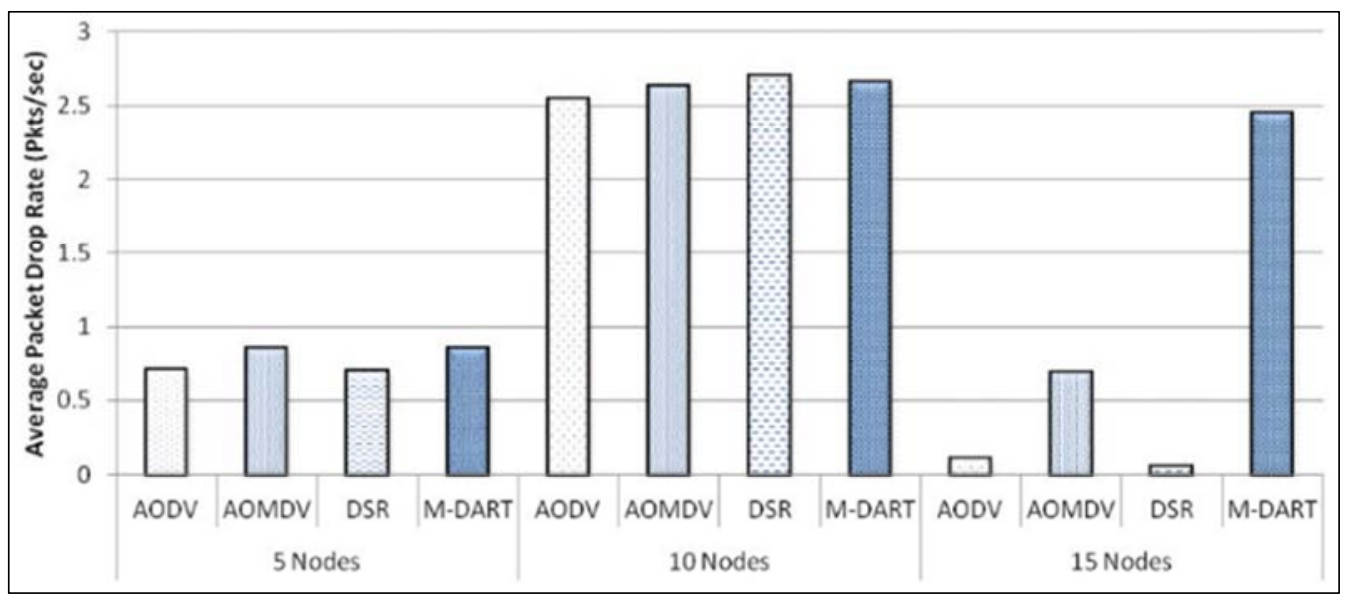

Figure 2. Average packet drop rate varying traffic scenario.

The average Delay per packet was analyzed by simulating the unipath (DSR, AODV) and multipath routing protocols (AOMDV, M-DART) while increasing the traffic sources. Figure 3 shows that the average delay per packet was lowest for AOMDV which means that the average delay per packet is lowest for multipath routing protocols as compared to unipath protocols. The reason came out that AOMDV used multiple, disjoint and loop-free paths for transmission to provide better network load balancing. The chances of multiple path availabilities increase with an increase in a number of nodes in a scenario. The delay will be less as packets are sent simultaneously through the multiple paths while sending data using multipath routing protocols.

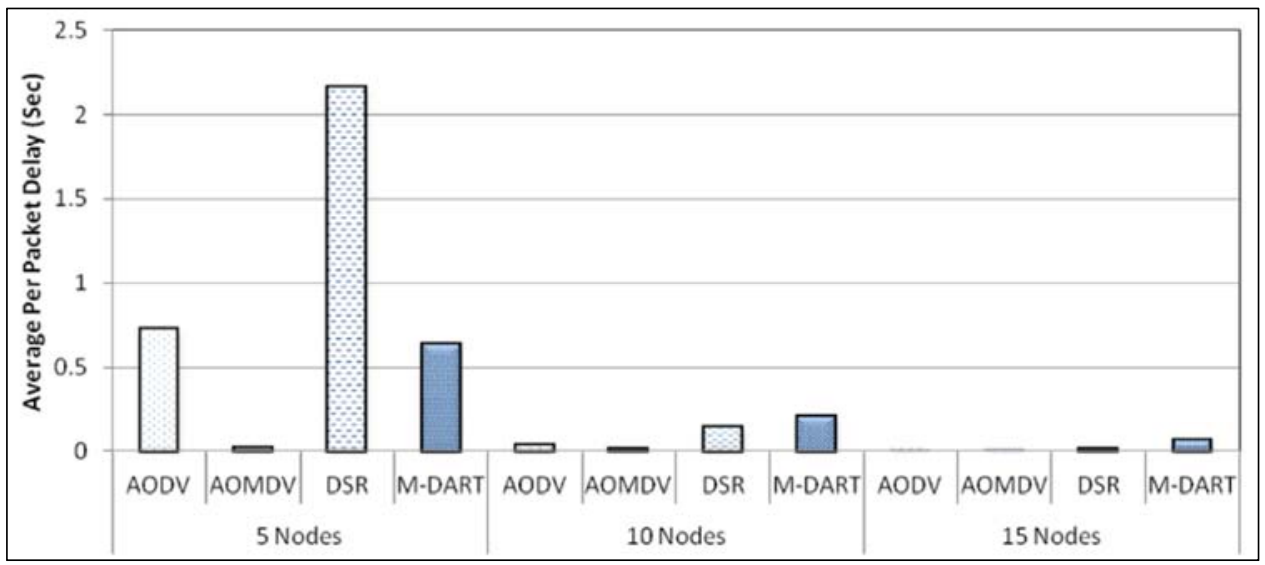

Figure 3. Average per packet delay in varying traffic scenario. 
The normal routing load is analyzed by varying the traffic source for comparing the performance of Unipath and multipath protocols. In figure 4 , Normal routing load for multipath routing protocols is higher than the unipath routing protocols in each sub scenario by 5, 10 and 15 nodes. Average Normal Routing Load for DSR is lowest and the routing load for M- DART is highest in most of the scenarios by comparing all the protocols, i.e. unipath and multipath routing protocols.

The normal routing load is lower for the Unipath routing protocol than the multi-path routing protocols because for maintaining multiple paths, multiple messages are also exchanged which increases the routing load. Routing, load increases, but at the same time number of paths for the exchange of data also increases, which is good for the performance of data efficiency. In this scenario, DSR has outperformed by having comparatively less average normal routing load due to its highly reactive nature of delivering packets in the presences of node movement or changes in other network conditions

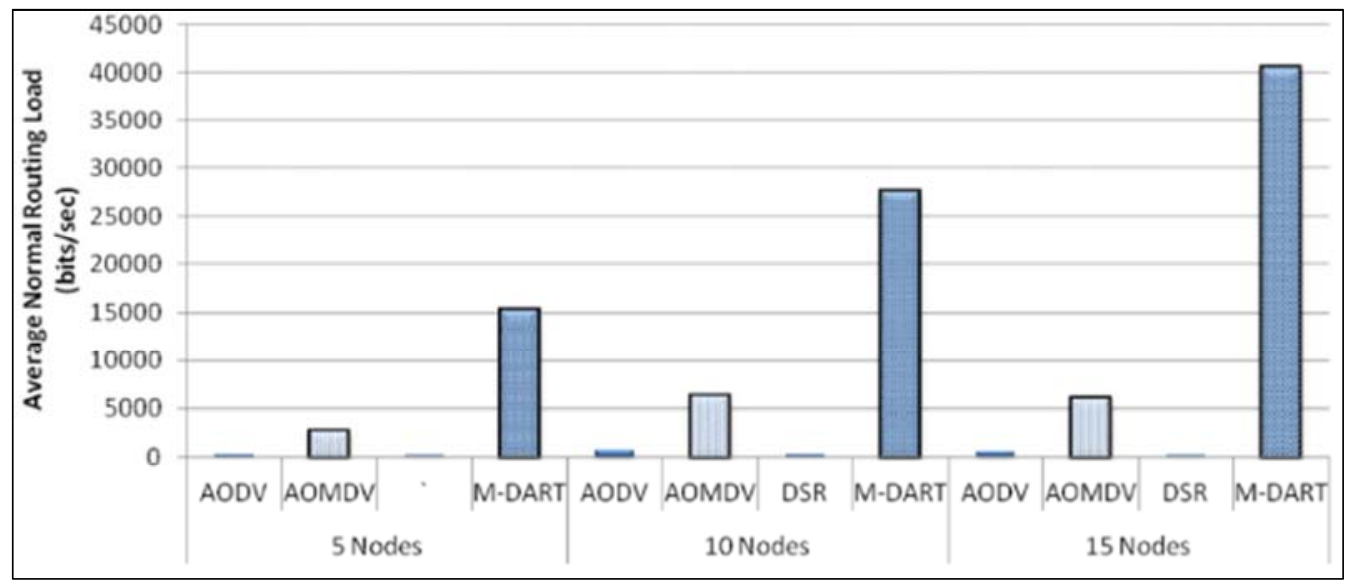

Figure 4. Average normal routing load in varying traffic scenario.

\subsection{Mobility}

Figure 5 shows that for analyzing Average Packet Drop Rate in each sub-scenario of varying mobility, i.e. $1.4 \mathrm{~m} / \mathrm{Sec}$, $3.3 \mathrm{~m} / \mathrm{Sec}, 11.11 \mathrm{~m} / \mathrm{Sec}$, it is observed that AODV has least packet dropped rate. As Topology changes due to Mobility and fact is that AODV in high mobility scenario responds quickly to link breakages and topological changes due to its convergence power. So, its observed that unipath protocols, i.e. DSR, AODV outperform multipath protocols, i.e. AOMDV, M-DART

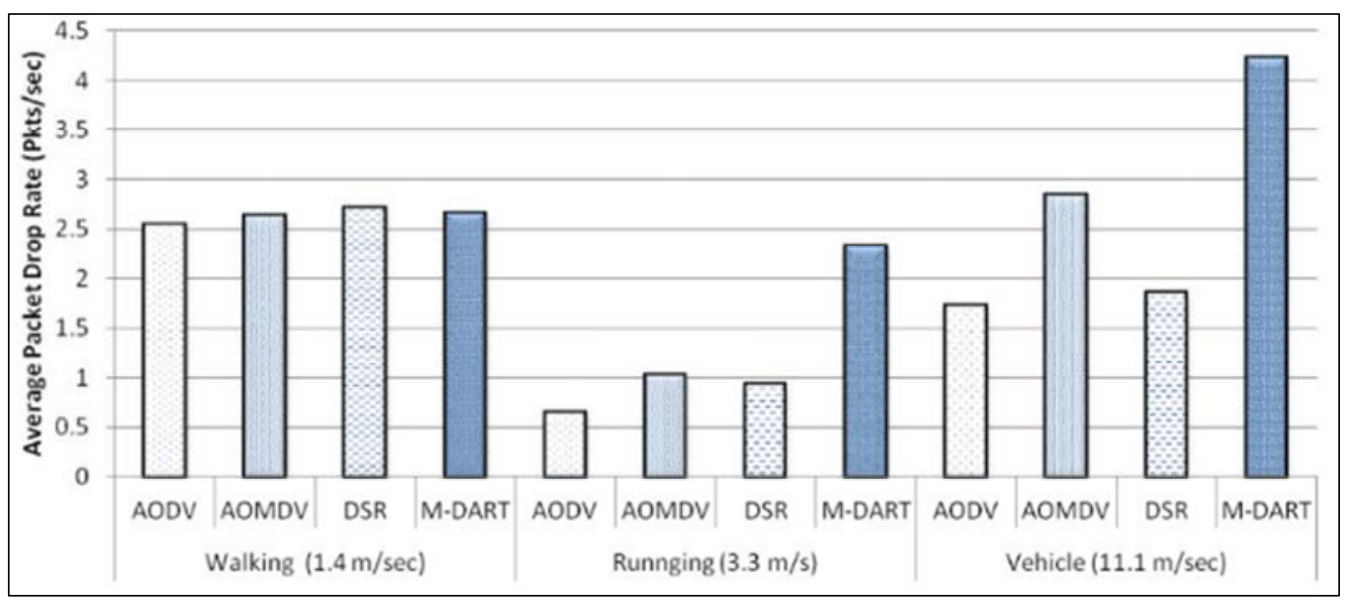

Figure 5. Average packet drop rate in varying mobility speed scenario.

The average delay per packet is observed in the scenario of varying mobility by comparing all the protocols of unipath and multipath. Figure 6 shows that AOMDV has least average delay per packet. Path failure rate increases with an increase in mobility speed. AOMDV used multiple paths to forward the packet on the alternate path without any extra delay.
Comparative analysis for unipath (DSR, AODV) and multipath protocols (AODV, M-DART) are used to analyze the parameter, i.e. Average Normal Routing Load in sending data from source to destination by varying mobility speed and keeping constant the nodes and pause time. Figure 7 shows that Average Normal Routing Load for DSR is low in all the protocols which means that unipath protocols show 
the best performance in this case of varying mobility. DSR has the lowest average normal routing load because in DSR, there are no periodic route advertisement, link status sensing or neighbour detection packets and also do not expect this information from underlying protocols.

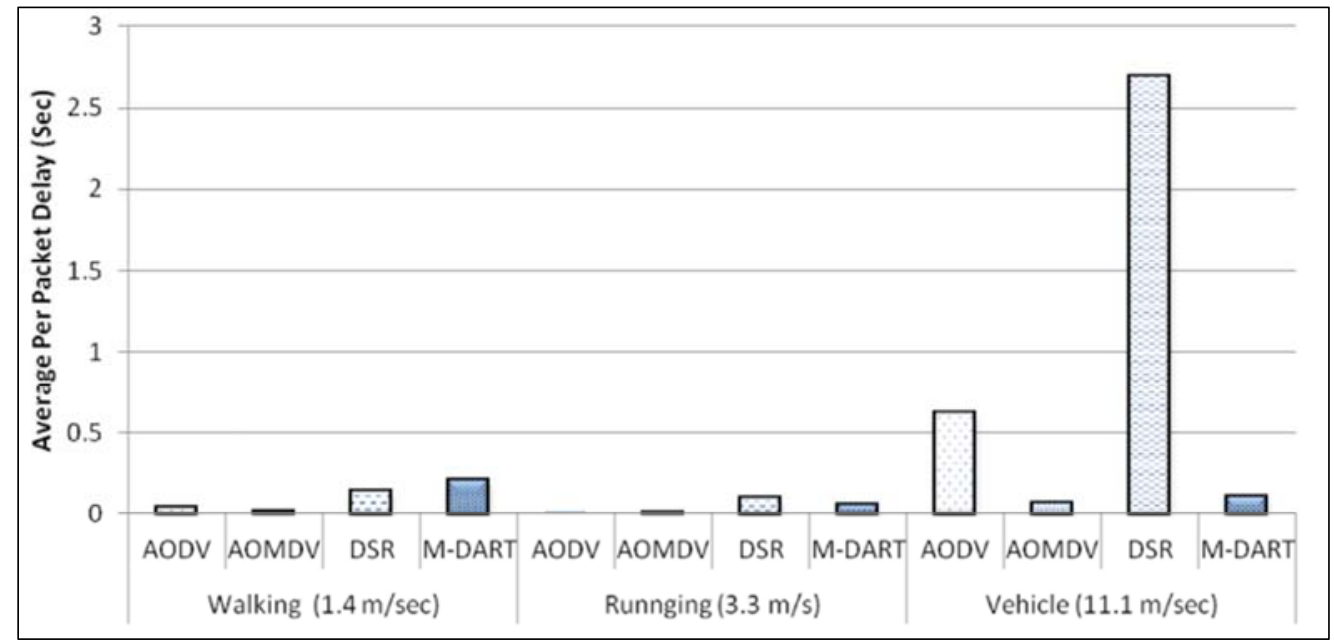

Figure 6. Average per packet delay in varying mobility speed scenario.

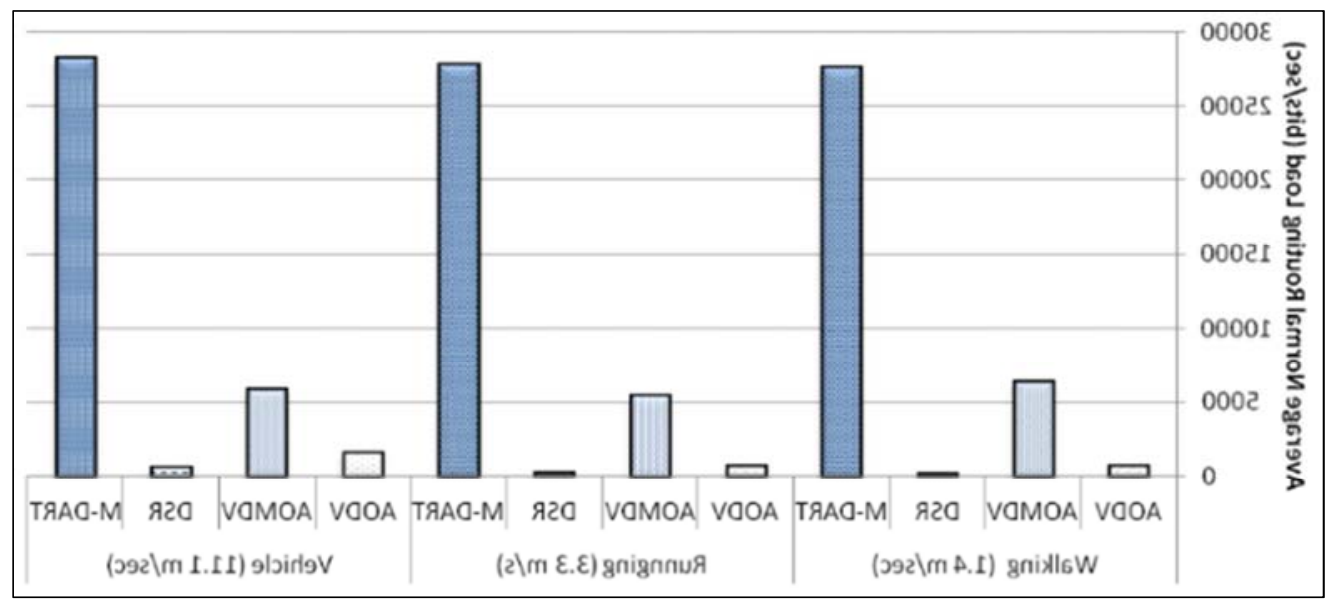

Figure 7. Average normal routing load in varying mobility speed scenario.

\subsection{Pause Time}

Figure 8 shows the graph of the average packet drop rate for all the protocols.

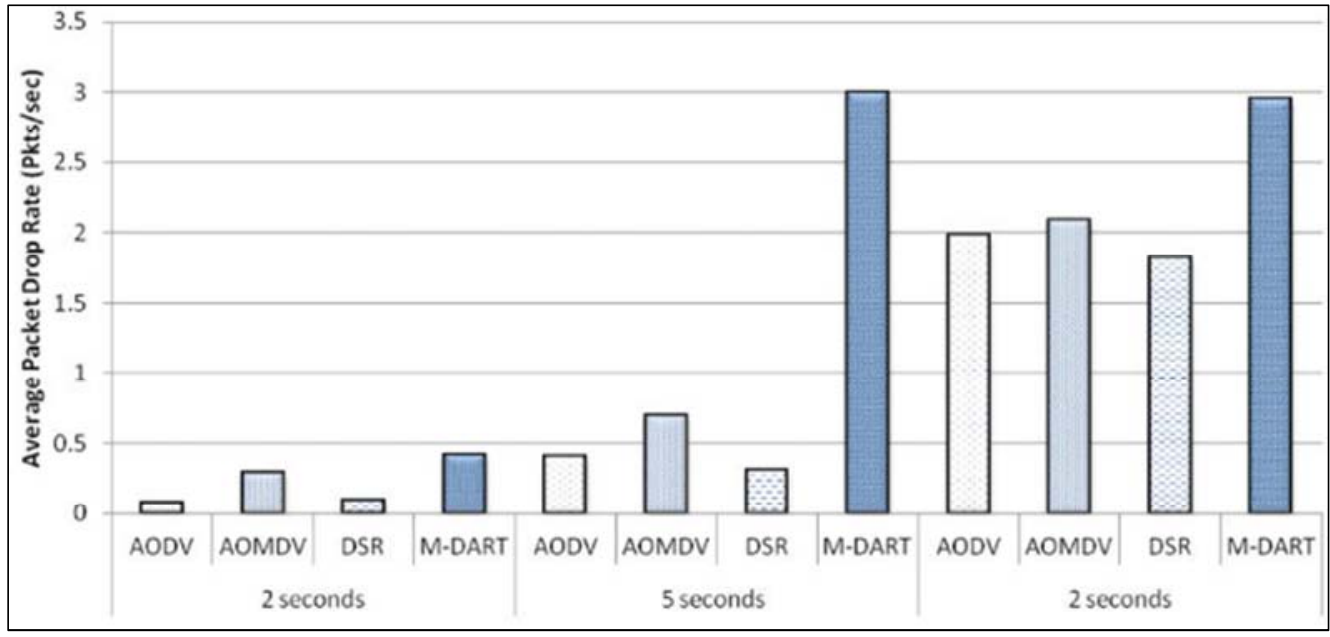

Figure 8. Average packet drop rate in the varying pause time scenario. 
Mostly average packet drop rate is lowest for DSR and highest for M-DART means summarizing Average Packet Drop Rate is low for Unipath but high for multipath protocols. When the pause time is $2 \mathrm{Sec}$, average packet drop rate is lowest for AODV, and highest for M-DART means it is low for Unipath but high for multipath protocols. When the pause time is $5 \mathrm{Sec}$, average packet drop rate is lowest for DSR and highest for M-DART means it is low for Unipath but high for multipath protocols. When the pause time is 10 $\mathrm{Sec}$, average packet drop rate is lowest for DSR, and highest for M-DART means it is low for Unipath but high for multipath protocols DSR trigger route discovery process when there is really need for route destination. In a static state, once the routes are discovered and established, they will be used for rest of communication. This decrease packet dropped rate in DSR to increase in pause time. Average Delay per Packet for each protocol is analyzed, and it is shown that AOMDV has least packet dropped rate which means that Average Delay per Packet is low for multipath and high for unipath as shown in Figure 9.

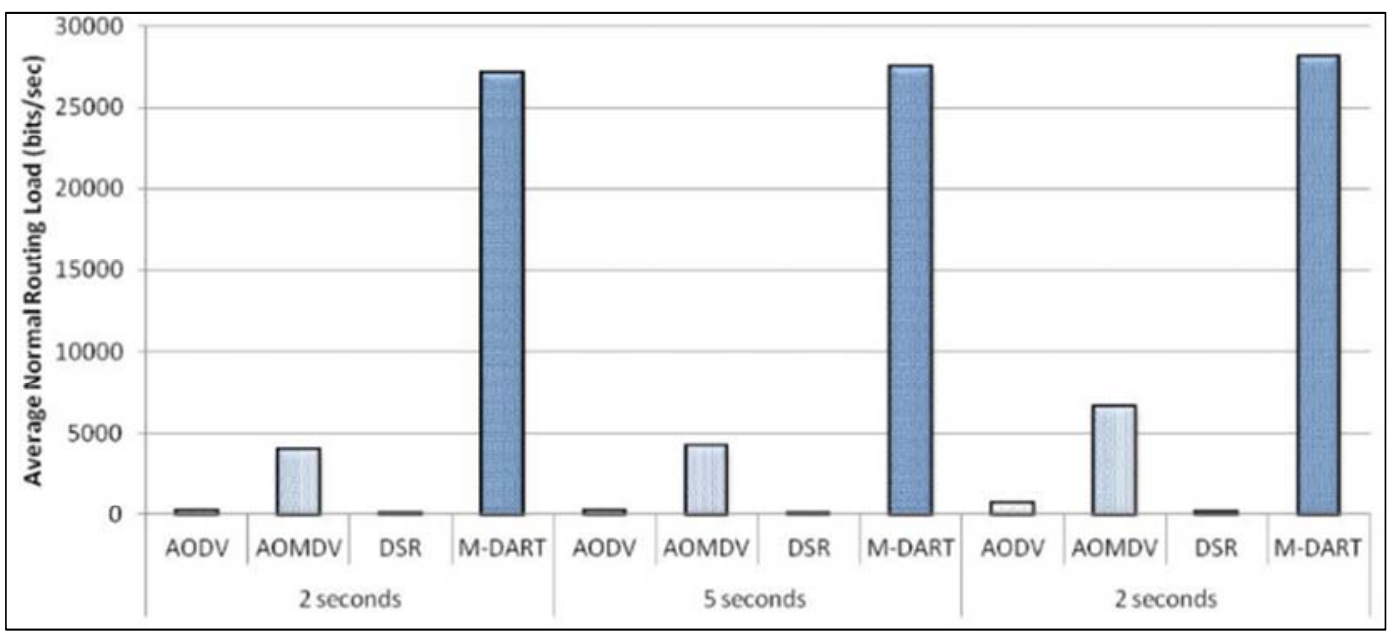

Figure 9. Average per packet delay in varying pause time scenario.

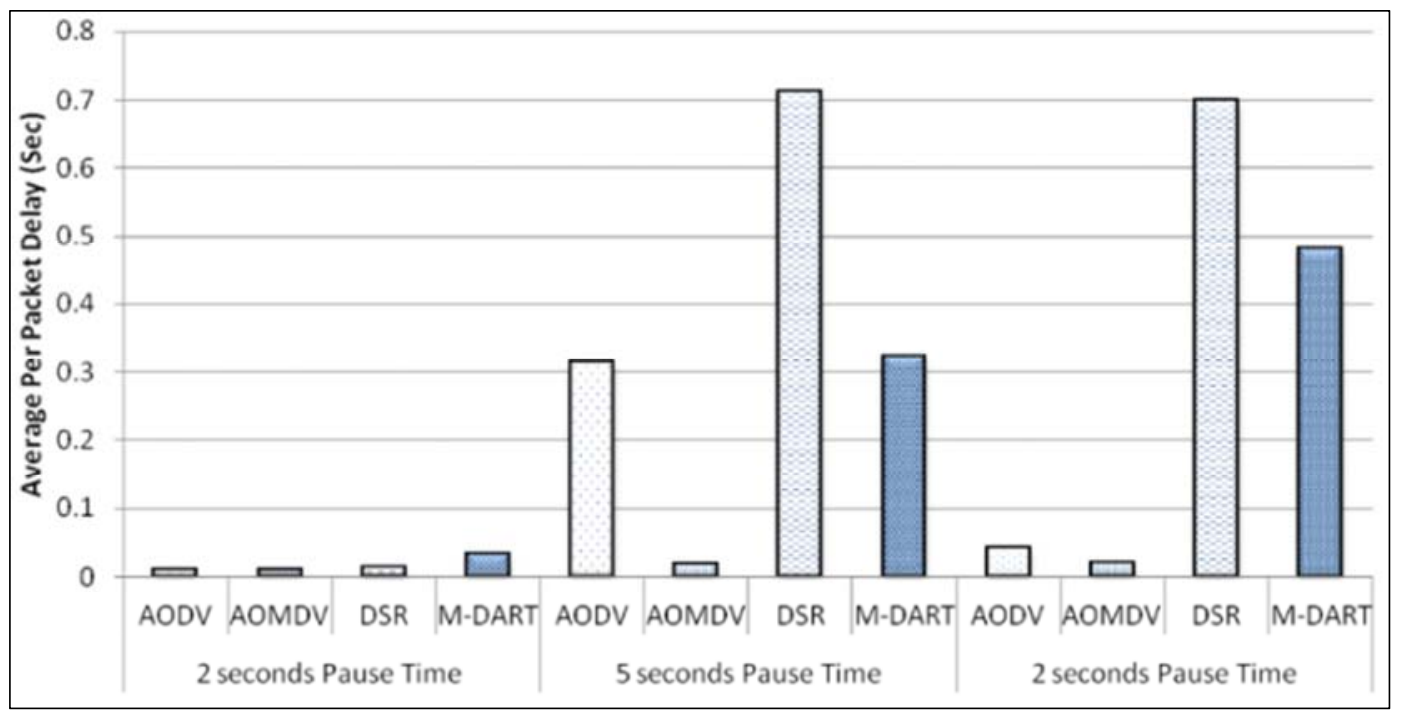

Figure 10. Average normal routing load in varying pause time scenario.

This is so because AOMDV has less average delay per packet with an increase in pause time due to loop-free, disjoint and multiple path effects. The packet is forwarded immediately on the alternate path to balance the network load and cope with network topology changes. In the scenario of varying pause time, the parameter, i.e. Average Normal Routing Load for sending data from source to destination by using each protocol in all the sub-scenarios, i.e. 2, 5 and 10
Sec. Figure 10 shows that DSR has least Average Normal Routing Load. In Pause time scenario, the DSR has lowest average routing overhead due to lack of periodic activities and pure on-demand nature. The nodes are more in a static position to increase in pause time. The routes need for communication are already established which results in a decrease in average normal routing load. 
Table 2. Percentage decrease of performance analysis parameters in all scenarios.

\begin{tabular}{|c|c|c|c|c|}
\hline Scenarios & Sub Scenarios & Average Packet Drop Rate & Average Packet Delay & Average Normal Routing Load \\
\hline \multirow{3}{*}{ Traffic Source } & 5 nodes & DSR $18.47 \%$ & AOMDV $99.07 \%$ & DSR $99.53 \%$ \\
\hline & 10 nodes & AODV $4.15 \%$ & AOMDV $90.97 \%$ & DSR $99.4 \%$ \\
\hline & 15 nodes & DSR $76.73 \%$ & AOMDV $81.25 \%$ & DSR $99.74 \%$ \\
\hline \multirow{3}{*}{ Mobility } & $1.4 \mathrm{~m} / \mathrm{s}$ & AODV $3.53 \%$ & AOMDV $93.78 \%$ & DSR $99.4 \%$ \\
\hline & $3.3 \mathrm{~m} / \mathrm{s}$ & AODV $71.95 \%$ & AODV $81.48 \%$ & DSR $99.28 \%$ \\
\hline & $11.11 \mathrm{~m} / \mathrm{s}$ & AODV 59.34\% & AOMDV $97.44 \%$ & DSR $97.83 \%$ \\
\hline \multirow{2}{*}{ Pause Time } & $2 \mathrm{Sec}$ & AODV $82.49 \%$ & AOMDV $23.08 \%$ & DSR $99.71 \%$ \\
\hline & $10 \mathrm{Sec}$ & DSR $38.24 \%$ & AOMDV $97.28 \%$ & $99.48 \%$ DSR \\
\hline
\end{tabular}

\section{Conclusion and Future Work}

By comparing unipath and multipath routing protocol, it is concluded that unipath routing has comparatively average packet drop rate (percentage decrease of $47.78 \%$ ) and average normal routing load (percentage decrease of $99.30 \%$ ). The multipath routing protocol has comparatively less average per packet delay (percentage decrease of $81.52 \%$ ). There is a single path for sending data in unipath routing protocols; the new path is selected when this path fails which causes a high end to end delay. In multipath routing protocol, multiple routes are used for data exchange and data is sent simultaneously through these paths which cause high packet drop rate and average normal routing load. Multiple routes are maintained at the cost of high normal routing load in multipath routing overhead.

\section{References}

[1] M. K. Marina and S. R. Das, "Ad hoc on-demand multipath distance vector routing," ACM SIGMOBILE Mobile Computing and Communi- cations Review, vol. 6, no. 3, pp. 92-93, 2002.

[2] L. Hanzo and R. Tafazolli, "A survey of QoS routing solutions for mobile ad hoc networks," Communications Surveys \& Tutorials, IEEE, vol. 9, no. 2, pp. 50-70, 2007.

[3] D. G. Andersen, A. C. Snoeren, and H. Balakrishnan, "Bestpath vs. multi-path overlay routing," in Proceedings of the 3rd ACM SIGCOMM conference on Internet measurement. ACM, 2003, pp. 91-100.

[4] N. S. Nandiraju, D. S. Nandiraju, and D. P. Agrawal, "Multipath routing in wireless mesh networks," in Mobile adhoc and sensor systems (MASS), 2006 IEEE international conference on. IEEE, 2006, pp. 741- 746.
[5] S. K. Gupta and R. Saket, "Performance metric comparison of aodv and dsdv routing protocols in manets using ns-2," International Journal of Research and Reviews in Applied Sciences, vol. 7, no. 3, pp. 339-350, 2011.

[6] A. Tuteja, R. Gujral, and S. Thalia, "Comparative performance analysis of dsdv, aodv and dsr routing protocols in manet using ns2," in Advances in Computer Engineering (ACE), 2010 International Conference on. IEEE, 2010, pp. 330-333.

[7] M. Z. Oo and M. Othman, "Performance comparisons of aomdv and olsr routing protocols for mobile ad hoc network," in Computer Engineering and Applications (ICCEA), 2010 Second International Conference on, vol. 1. IEEE, 2010, pp. 129-133.

[8] G. Singh, D. S. Gupta, and S. Singh, "Performance evaluation of dht based multi-path routing protocol for manets," International Journal of Scientific and Research Publication, vol. 2, no. 6, 2012.

[9] A. K. Gupta, H. Sadawarti, and A. K. Verma, "Performance analysis of aodv, dsr \& tora routing protocols," International Journal of Engineering and Technology, vol. 2, no. 2, p. 226, 2010 .

[10] W. Kiess and M. Mauve, "A survey on real-world implementations of mobile ad-hoc networks," Ad Hoc Networks, vol. 5, no. 3, pp. 324-339, 2007.

[11] Hasan, M. Z., Al-Rizzo, H., \& Al-Turjman, F. (2017). A Survey on Multipath Routing Protocols for QoS Assurances in Real-Time Wireless Multimedia Sensor Networks. IEEE Communications Surveys \& Tutorials.

[12] Mallapur, S. V., Patil, S. R., \& Agarkhed, J. V. (2017). Load Balancing Technique for Congestion Control Multipath Routing Protocol in MANETs. Wireless Personal Communications, 92 (2), 749-770.

[13] Bondre, V., \& Dorle, S. (2017). Performance Analysis of AOMDV and AODV Routing Protocol for Emergency Services in VANET. European Journal of Advances in Engineering and Technology, 4 (4), 242-248. 